\title{
Unravelling Molecular Genetics of Gallbladder Cancer: Implications for Targeted Therapy
}

\author{
Mahendra K Singh ${ }^{1}$ and Hemant K Bid ${ }^{2 *}$ \\ ${ }^{1}$ Department of Surgery, University of Miami Miller School of Medicine, USA \\ ${ }^{2}$ Resonant Therapeutics, Ann Arbor, USA
}

Submission: July 18, 2017; Published: July 27, 2017

*Corresponding author: Hemant K Bid, Resonant Therapeutics, Inc, Ann Arbor, Michigan, USA Email: hbid@resonantrx.com

\begin{abstract}
Abbreviations: GBC: Gallbladder Cancer; PCR: Polymerase Chain Reaction; SSCP: Single Strand Conformation Polymorphism; MSI: Microsatellite Instability; CIS: Carcinoma In Situ; AJPBD: Anomalous Junction of Pancreatico Biliary Duct; FHIT: Fragile Histidine Triad; CC: Chronic Cholecystitis; XGC: Xanthogranulomatous Cholecystitis; SAGE: Serial Analysis of Gene Expression; CTGF: Connective Tissue Growth Factor; CLIC1: Chloride Intracellular Channel 1; NAT2: N-acetyl Transferase2; GSTM1: Glutathione S-transferase Class Mu; CYP1A1: Cytochrome P450 1A1; TNFA : Tumor Necrosis Factor Alpha; CYP7A1: Cholesterol 7 Alpha-Hydroxylase; EGFR: Epidermal Growth Factor Receptor
\end{abstract}

\section{Introduction}

Gallbladder cancer (GBC) is a rare but highly aggressive neoplasm with a dismal prognosis and a median survival of less than one year in the locally advanced or metastatic setting. About $90 \%$ of patients are detected at advanced stages, and systemic chemotherapy is the mainstay of their treatment. Among the few lucky ones who could undergo curative resection the recurrence rates are high. The treatment options for these patients are limited and multiple modalities of therapy from targeted therapy to immunotherapy and combination therapies have been tested in this disease. Targeted therapies have failed to show a survival benefit. An understanding to the relationship between molecular genetics and pathogenesis of gallbladder cancer can add new insights to understanding of signaling pathways that may be targeted by therapeutic agents. In this article, we will review recent update regarding molecular epidemiology, pathogenesis, and molecular genetics of gallbladder cancer.

Gallbladder cancer (GBC) being quite uncommon disease in the west, where most of the advancement in molecular genetics took place, it is not surprising that serious efforts to uncover the mechanistic insights of GBC at molecular level started very late. However, reports implicating role of genetic/hereditary predisposition can be traced in scientific literature as early as in early 80 s Trajber et al. [1], Weiss et al. [2]. Late 70s and early 80s was an exciting time in biological research especially for cancer researchers who were establishing the links between cancer and heredity and role of mutated genes were beginning to be discovered with regard to various types of cancers. It was 1983 when Hecht and his colleagues who used karyotyping techniques and first reported a high degree of aneuploidy including multiple instances of missing chromosomes, extra chromosomes, and chromosome rearrangements in tumor tissue specimen obtained from a Papago Indian woman with gallbladder adenocarcinoma in the United States. Double minute chromosomes and homogeneous staining regions on chromosomes were also reported to be present in the cancer cells Hecht et al. [3].

First few important studies demonstrating familial predisposition of gallbladder cancer include a report from Japan in which the authors presented two cases of anomalous pancreaticobiliary duct union that occurred in a mother and her daughter; the former was associated with gallbladder adenocarcinoma and the latter, gallbladder adenoma with high grade dysplasia Miyazaki et al. [4]. However, it was Tsuda and his colleagues who first used molecular methods in 1989 to detect genetic changes at molecular level in gallbladder cancer tissues Tsuda et al. [5]. They performed Southern blot analyses on squamous cell carcinoma of gallbladder, using a panel of several oncogenes as probes.

Two genes HST1 and INT2 genes, both of which were mapped to chromosome 11 at band $\mathrm{q} 13$, were found to be 
significantly amplified together in a squamous cell carcinoma of the gallbladder. Although it is not very significant study as squamous cell carcinomas of gallbladders are not so common as opposed to adenocarcinomas, however, this observation prompted geneticists/molecular biologists for further molecular based studies in gallbladder malignancies. Another Japanese study published in 1990, first analyzed point mutations at K-ras codon 12 using polymerase chain reaction (PCR) and a direct sequencing method, however, no mutations were detected in all of 9 gallbladder carcinomas Tada et al. [6]. Later in 1993, Kamel et al. [7] first time reported the co-expression of mutant form of tumor suppressor gene p53 and c-erbB-2 in GBC using immunohistochemistry and suggested that alterations of these genes might act in concert in the malignant transformation of gall bladder epithelial cells. They also suggested that the occurrence of p53 expression in gall bladder dysplasias implicated that p53 mutations could be an early event in the evolution of some gall bladder carcinomas, as had been suggested for some other types of tumors, such as lung squamous cell carcinomas Kamel et al. [7].

A group of researchers from Chile, another geographical area which is known for its high GBC incidence, reported its first molecular study that analyzed the DNA content in gallbladder carcinoma and its relation to histological and cytological features Roa et al. [8]. Abnormal DNA content such as diploid DNA content, and aneuploidy was observed in little more than half of the patients (48 (51\%) of 95 cases) studied with GBC which were analyzed by flow cytometric study. Abnormality in DNA content was also found to be associated with late stages of disease. Another small but an important study by Japanese researchers in following year, first time demonstrated mutations in p53 gene using a molecular technique, PCR-single strand conformation polymorphism (SSCP) in almost one third of the patients with GBC Takagi et al. [9]. Following several years saw an increasing number of published studies mostly from Japanese investigators observing mutations in K-ras Watanabe et al. [10], Hanada et al. [11], Matsubara et al. [12], Tomono et al. [13], Saetta et al. [14], Iwase et al. [15], Tanno et al. [16] and p53 genes Hanada et al. [11], Ajiki et al. [17], Fujii et al. [18], Hanada et al. [19], Jonas et al. [20], Yokoyama et al. [21] in significant percentage of patients with GBC.

By late 1990s, many studies had detected the mutations and/or the over-expression of mutated oncogenes and tumor suppressor genes in GBC, however, most of them lacked the mechanistic explanations of their findings. In 1999, first important study was published discussing the molecular mechanism underlying tumorigenesis of gallbladder carcinomas Wistuba et al. [22] in which researchers pointed out that although most gallbladder carcinomas evolved from dysplasia and carcinoma in situ, the role of gallbladder adenomas in the pathogenesis of gallbladder carcinoma was still controversial. To determine whether the molecular abnormalities of adenomas are similar to those found in carcinomas, they obtained extracted DNA from precisely micro dissected tissue from 16 gallbladder adenomas (14 pyloric and 2 intestinal-type). They determined the presence of mutations in TP53, K- and N-ras genes, and loss of heterozygosity ( $\mathrm{LOH})$ at five other chromosomal regions (5q22 APC-MCC region, RB, TP53, DCC and 9p21-CDKN2a) which are known to have frequent genetic aberration in many other cancers.

Based on their findings these researchers concluded that gallbladder adenoma lacked the molecular changes frequently detected in dysplasia, carcinoma in situ, and invasive carcinoma of the gallbladder. Likewise the occurrence of K-ras mutations at codon 12 and 61 in $25 \%$ of adenomas strongly suggested that these lesions were not precursors of invasive gallbladder carcinoma Wistuba et al. [23]. Later in 2001, a Japanese study confirmed this hypothesis by analyzing beta-catenin protein expression and mutations in corresponding gene, which is a regulator of cell-cell adhesion and intranuclear transcription, in gallbladder carcinogenesis. Authors observed that the gallbladder adenomas showed significantly stronger expression of beta-catenin protein in the cytoplasm and the nucleus than carcinomas ( $\mathrm{P}<0.05$ and $\mathrm{P}<0.001$, respectively), and exon 3 mutations in beta-catenin gene were observed in $62.5 \%$ (10 of 16) of adenomas, but only 4.8\% (1 of 21) of carcinomas. Using beta-catenin as a molecular marker, these investigators demonstrated the adenoma-carcinoma sequence as a minor pathway in gallbladder carcinogenesis Yanagisawa et al. [24].

Besides K-ras and p53, role of some other cancer associated proteins were also explored in GBC. In a study from Japan, expression of mucin core protein MUC1 and MUC2 was examined at the protein and mRNA level in 55 cases of carcinoma and 20 of dysplasia, and in 15 non-dysplastic epithelia of the gall bladder Yamato et al. [25]. Based on their observation they suggested that MUC1 expression by gall bladder carcinoma reflected histological dedifferentiation, increased proliferative activity, and invasiveness, while MUC2 expression was related to lower proliferative activity and reflected some differentiation towards goblet cells; and that MUC1 expression in gall bladder dysplasia also reflected malignant transformation Yamato et al. [25]. Same year a Korean study revealed some molecular alterations in the carcinogenesis of gallbladder carcinoma by analyzing 32 carcinoma cases and 11 dysplasia cases of gallbladder for LOH and microsatellite instability (MSI) on chromosomal regions 3p, 5q, 8p, 9p, 13q, 17p, and 18q with 17 microsatellite markers Chang et al. [26]. They observed that $\mathrm{LOH}$ on $5 q$ was an early change of carcinogenesis in gallbladder and that $\mathrm{LOH}$ on $3 p$ and $9 p$ was related to the progression of gallbladder carcinoma $\mathrm{LOH}$ on $13 \mathrm{q}$ and $18 \mathrm{q}$ was likely to be a late event. LOH on $17 p$ occurs not only in dysplasia but it was also found to be increased during the subsequent tumor stages. They demonstrated that while accumulation of $\mathrm{LOH}$ was associated with carcinogenesis of the gallbladder, role of MI was not 
significant Chang et al. [26]. A subsequent Japanese study aimed to investigate the genetic changes in gallbladder carcinogenesis suggested that $\mathrm{LOH}$ on $17 \mathrm{p}$ may played an important role in the evolution of GBC from a relatively early phase, while LOH on $9 p$ and 18q may played roles in progression Hidaka et al. [27]. Another study from Japan, however, emphasizing the etiologic context of pancreaticobiliary malunion (which is a major and uniquely associated factor in Japanese patients with GBC), hypothesized that epithelial hyperplasia played an important role in gallbladder carcinogenesis and based on their findings they believed that neoplastic development in gallbladder with this anatomic anomaly also evolved through a multistep process associated with hyperproliferation and genetic alterations Obara et al. [28].

By the end of 1990s, majority of researchers working in this field, considered that while TP53 (17p13) and p16(Ink4)/ CDKN2 (9p21-22) gene loci abnormalities were frequent and early events in the pathogenesis of this neoplasm, deletions at other chromosomal regions (8p21 and DCC gene at 18q21 loci) played an important role in the development of GBC. K-ras gene mutations appeared to be an infrequent event in this neoplasm, except in gallbladder carcinomas associated with congenital abnormalities of the biliary tract. Genetic studies confirmed that the sequence dysplasia-carcinoma in situ (CIS) is the usual route for the development of gallbladder carcinoma Wistuba \& Albores-Saavedra [23]. Another study in following year from Japan analyzed 30 gallbladder carcinomas to investigate the role of genetic alterations in their tumorigenesis, and to study correlations with their clinicopathological features.

Using multiple markers to study MSI and $\mathrm{LOH}$, authors observed that there were two independent genetic pathways in gallbladder carcinogenesis; one dependent on MSI pathway and another on LOH pathway Yoshida et al. [29]. None of the patients with MSI-positive tumors showed lymph node metastasis, and there was an inverse correlation between MSI and the presence of $\mathrm{LOH}$ in gallbladder carcinoma. Soon, a Korean study demonstrated that multiple $\mathrm{LOH}$ were associated with the development of dysplasia and the malignant transformation of GBC and genetic alterations of K-ras, p53, and p16 were important steps in the malignant changes of dysplasia while MSI had only a limited role in GB cancer development Kim et al. [30]. However, a report from Chile published in 2005 emphasized the role of MSI in GBC Roa et al. [31]. This group observed MSI in equal proportions in early and late cancers of gallbladder. They demonstrated that MSI was found in premalignant lesions too and indicated that inactivation of mismatch repair genes such as hMLH1, hMSH2, and hMSH6 occurred early in gallbladder carcinogenesis. However, contradicting reports on the role of MSI in GBC kept emerging from different parts of the world. A Greek study in 2006 again asserted the minor role of MSI in GBC Saetta et al. [32]. However, it is important to note that these differences in results could be due to selection of various markers to study
MSI in different studies; however role of different mechanisms especially in context of different etiologic factors underlying the events in tumorigenesis in different populations of the patients is certainly a possibility.

Availability of newer and more effective molecular methods in early years of 21st century, made it possible for gallbladder researchers to conduct more studies using sophisticated analyses to detect molecular alterations in gallbladder carcinogenesis. While a Japanese study using allelotyping analysis demonstrated that gallbladder carcinoma associated with anomalous junction of pancreaticobiliary duct (AJPBD), a genetic anatomic anomaly, had high frequent allelic loss and had two new regions which they believed might harbor putative tumor suppressor genes Nakayama et al. [33], investigators based in USA (where GBC is a rare malignancy) developed their interest in studying this intriguing disease and used a more sophisticated and global analytical technique, genome-wide allelotyping analysis to reveal multiple sites of allelic loss in GBC and reported at least 21 chromosomal regions with frequent allele losses to be involved, suggesting that several putative tumor suppressor genes were inactivated in its pathogenesis Wistuba et al. [34]. These data were the first in its kind which provided global estimates of the extent of genetic changes leading to GBC and have potential for the identification of new tumor suppressor genes and multiple new markers for translational research. A Greek study also appeared in the same year which demonstrated that p53 alterations were more likely to take part in the de novo pathway of gallbladder carcinogenesis Saetta et al. [35].

One of the most significant studies reporting other aberrated molecular lesions (besides p53 and K-ras) in GBC resulted from an collaborative effort between Chile and US based researchers where investigators Wistuba et al. [36] found out that the fragile histidine triad (FHIT) candidate tumor-suppressor gene located at $3 \mathrm{p} 14.2$, one of the putative tumor suppressor genes, was involved in the pathogenesis of this highly malignant neoplasm. Much later in 2009, another US based study demonstrated the loss of FHIT and Wwox (another fragile gene product) expression was part of an early event in the pathogenesis of GBC Bloomston et al. [37].

Later in the same year, our own group (senior author of this chapter) based in Lucknow, India, analyzed LOH and MSI in FHIT gene and p53 expression in GBC, Chronic Cholecystitis (CC), Xanthogranulomatous Cholecystitis (XGC), and normal GB to elucidate the role of FHIT gene in GBC by using five microsatellite markers D3S1217, D3S1300, D3S1313, D3S1600, and D3S2757. We observed the frequency of MSI and LOH was $17.5 \%$ and $27.5 \%$ respectively among GBC cases and noted significant difference between GBC and normal GB ( $p=0.02$ ), and GBC and CC groups $(p=0.002)$ when LOH was compared. Our results also suggested that CC might act as a preinvasive stage in the pathogenesis of GBC Priya et al. [38]. XGC, a chronic inflammatory disease of the gallbladder, a variant of the chronic 
cholecystitis, is believed to be a precursor state of GBC by some groups of gallbladder researchers. In a smaller but significant study, a group of Japanese researchers attempted to solve the puzzle of XGC at molecular level Takada et al. [39]. However, this analysis confirmed that while etiopathologic factors of XGC might have relation with cancer, but XGC itself may not be the direct cause for cancer Takada et al. [39].

An Italian group studied the MSI status and p53 expression in a series of $71 \mathrm{GBC}$ of different histologic type. All neoplasms were examined combining a microsatellite analysis at mononucleotide locus BAT-26 and an immunohistochemical study for hMSH2, hMLH1, and p53 proteins and markers of gastric and intestinal differentiation. They demonstrated that MSI does not play a role in the development of GBC while p53 seems to be the most important alteration found in a large proportion of these cancers, with the only exception of mucinous and squamous GBC Sessa et al. [40]. Same year, a small study conducted at John Hopkins Medical Institute, demonstrated that the acquisition of hypermethylation at multiple tumor-suppressor gene-promoter sites might contribute to tumor formation and progression within the chronically inflamed gallbladder. Since this study included a large cohorts of specimens obtained from two different populations, Chile and United States, they also observed the apparent differences in methylation patterns among the Chilean and US gallbladder cases and hypothesized that these differences may indicate a unique biology associated with this cancer in different parts of the world House et al. [41].

In a subsequent Chilean study, mutations in the mitochondrial DNA (mtDNA) had also been observed in GBC, suggesting mtDNA mutations should be additionally investigated in GBC pathogenesis in patients from different geographical regions so as to be included in a panel of molecular biomarkers for GBC early detection strategy Tang et al. [42]. Same year, John Hopkins based investigators conducted a study which constituted the most comprehensive methylation profiling in GBC and demonstrates that this neoplasm had a distinct pattern of abnormal gene methylation. Whereas gallbladders from healthy individual were not available, their finding of methylation in CC cases without cancer suggested that this phenomenon represented an early event in the pathogenesis of GBC Takahashi et al. [43].

The high frequency of gene methylation of the gene promoter area for the CDKN2A (p16), FHIT, APC, and CDH1 genes was again observed by a Chilean group which suggested that the inactivation of tumor suppressor genes and of the genes related to the control of cellular proliferation through this mechanism was involved in gallbladder carcinogenesis Roa et al. [44]. Another Chilean study confirmed that epigenetic inactivation by abnormal promoter methylation was a frequent event in chromosome $3 p$ candidate tumor suppressor genes such as DUTT1 (3p12), FHIT (3p14.2), BLU, RASSF1A, SEMA3B and hMLH1 (3p21.3) in GBC pathogenesis, with more pronounced hypermethylation in genes two genes, SEMA3B (3p21.3) and FHIT (3p14.2) Riquelme et al. [45].

By the mid 2000s, many reports were published regarding mutations in K-ras genes and/or overexpression of this oncogene in GBC, a small study from Greece also reported mutations in B-raf gene, an important component of Ras signaling pathway (RAS/RAF/MEK/ERK). They reported that almost one third of all studied patients with GBC had mutations in B-raf gene. It is important to note that Ras pathway is organized as a cascade in which RAF proteins are activated in a RAS-dependent manner. Considering it is known that the RAS signaling pathway can be activated by mutation at various levels including B-raf, it was an important finding re-emphasizing the role of Ras pathway even in those GBC cases where no mutation was detected in K-ras gene Saetta et al. [46]. Our own group also demonstrated a significant number of patients with GBC treated at a North India based hospital, had mutation in codon 12 of the K-ras oncogene Singh et al. [47].

While this study was first of its kind from India using molecular methods to detect changes at molecular level in an oncogene, it also demonstrated that detection of such molecular aberration in an oncogene could be performed on as small amount of specimens as obtained from fine-needle aspirates, and it may also be useful as an adjunct to cytological examination in making definitve diagnosis in patients with other risk factors at least in a sub-set of patients harboring K-ras mutations as part of the early tumorigenesis of gallbladder. This study also suggested that chronic inflammation may play an etiologic role in gallbladder carcinogenesis Singh et al. [48]. However, with growing realization of cancer being a much more complex disease than it was thought earlier, cancer researchers working on GBC, initiated pathway/mechanism oriented studies.

One of such study conducted in Japan suggested that homozygous deletion, a combination of $\mathrm{LOH}$ and promoter hypermethylation, and multiple $\mathrm{LOH}$ are major mechanisms of p16 inactivation in GBC Tadokoro et al. [49]. Another such study to understand geographic differences in genetic changes involved in gallbladder carcinogenesis between two distinct high-incidence areas of Japan and Hungary, specimens obtained from two populations based in these two countries was conducted in a collaborative effort. This study demonstrated that the vast differences in genetic alterations between two populations with GBC such as high MSI was reported in majority of Japanese patients as opposed to only one patient from Hungary showed high-MSI. This clearly demonstrated that geographic variation could play significant role in the process of gallbladder carcinogenesis at mechanistic level Nagahashi et al. [50].

In a US based study relying on an state-of-art technique, serial analysis of gene expression (SAGE) analysis, investigators generated SAGE libraries from three stage-matched GBC patients (representing Hispanic/Latino, Native American, and 
Caucasian ethnicities, respectively) and one histologically alithiasic gallbladder. Real-time quantitative PCR analysis was used on micro dissected epithelium from five matched GBC and corresponding nonneoplastic gallbladder mucosa and Immunohistochemical analysis was also performed on a panel of 182 archival GBC in high-throughput tissue microarray format to understand the complexity of the GBC. From a unbiased analysis of the GBC transcriptome by SAGE, this study identified connective tissue growth factor (CTGF) expression as a predictive biomarker of favorable prognosis in this malignancy. These SAGE libraries from GBC and nonneoplastic gallbladder mucosa are publicly available at the Cancer Genome Anatomy Project web site and should facilitate much needed research into this lethal neoplasm Alvarez et al. [51].

In a small attempt to identify metastasis-associated proteins in GBC as advanced gallbladder cancer has an extremely poor prognosis because most of them are already metastasized by the time when they are diagnosed, a Chinese study demonstrated that overexpression of CLIC1 promoted cell motility and invasion of GBC-SD18L in vitro, while RNA interference of CLIC1 remarkably decreased cell motility and invasive potency of GBC-SD18H in vitro, indicating that chloride intracellular channel 1 (CLIC1) might play an important role in metastasis of GBC. This was a first study to identify a regulator of metastasis in GBC Wang et al. [52]. Another wide-genome analysis based study conducted at Memorial Sloan-Kettering Cancer Center, New York, US, revealed that short segments of chromosomes $1 p, 3 p, 6 q, 8 p, 9 p$, and $14 q$ were commonly deleted regions and most commonly amplified regions included segments of 1q, 3q, 5p, 7p, 7q, 8q, and 20q in GBC Miller et al. [53].

An interesting finding about GBC have been reported by an Indian group of investigators in which the genomic instability in the E-cadherin ( $\mathrm{CDH} 1$ ) gene and correlated the findings with its protein expression in GBC and in other gall bladder diseases viz. chronic cholecystitis (CC), xantho-granulomatous cholecystitis (XGC), and normal gallbladder to explicate its role in GBC tumorigenesis. MSI and $\mathrm{LOH}$ in $\mathrm{CDH} 1$ were studied using D16S421, D16S496, D16S503, D16S512, D16S2624, and D16S3021 microsatellite markers and D2S123 (2p16), D2S382 (2q24), D6S292 (6q21-23), D7S480 (7q31), and D17S796 (17p13.1-3) were used to investigate genomic instability at $2 p$, $2 q, 6 q, 7 q$, and $17 p$ loci in specimens obtained from GBC, CC, $\mathrm{XGC}$, and normal gallbladder tissues.

IHC analyses were also carried out to analyze the E-cadherin and p53 protein expression. They observed a high LOH in CDH1 and other markers in GBC and XGC as compared to CC; however, it did not correlate with its protein expression in GBC cases. Loss of E-cadherin expression was high in GBC (67\%), while majority of the CC (94\%) and XGC (91\%) cases retained positive E-cadherin expression. Overexpression of p53 was high in GBC (43\%) whereas CC, XGC, normal GB cases were negative for p53 overexpression. None of the normal GB cases showed genomic instability at any of the markers. High LOH in CDH1 and other chromosomal loci in GBC suggested that the genomic instability followed a $\mathrm{GBC}>\mathrm{XGC}>\mathrm{CC}$ trend during the process of neoplastic transformation in $\mathrm{GB}$, highlighting the fact that $\mathrm{CC}$ might act as a precursor lesion of GBC Priya et al. [54].

\section{Genetic Polymorphism and Risk of GBC}

In the early 2000 s, published evidences were accumulating in support of the theory that the genetically determined risk factors might considerably contribute to the development of neoplastic diseases. It is believed that they may result, among others, from the differences in the metabolism of environmental carcinogens and mechanisms of DNA repair and the kind and rate of metabolism is genetically determined by polymorphic enzyme coding genes participating in the process of xenobiotic transformation. Genetic polymorphism has been confirmed for a number of enzymes involved in the reaction of oxidation or conjugation of exo- and endogenous xenobioties.

Gene variability may alter the expression or enzymatic activity of coded enzymes. First such study with relations to GBC was conducted in Japan in which authors investigated the relation between cytochrome P4501A1 (CYP1A1) gene polymorphisms and the risk of GBC. To clarify individual differences in susceptibility to gallbladder carcinogenesis, they examined the frequency and relationship between the CYP1A1 polymorphisms and the development of GBC. They demonstrated that females with genotypes C and/or Ile/Val in CYP1A1 gene might have a high genetic susceptibility to the development of gallbladder cancer Tsuchiya et al. [55]. Next was our own study that was performed on a large cohort of 153 patients with GBC from North India.

We reported that the frequency of the $\mathrm{X}$ - allele of apolipoprotein B (apoB), a gene which had previously been reported to be associated with altered serum lipids and susceptibility to cholesterol gallstones (GS), was significantly increased in GBC patients with or without GS (odds ratio=2.3 and 1.7 , respectively), which suggested that the apoB-XbaI gene polymorphism confers susceptibility to carcinoma of the gallbladder under specific environmental conditions Singh et al. [48]. A collaborative study between Japanese and Hungarian groups much later confirmed that Val allele of cytochrome P4501A1 (CYP1A1) gene may contribute to the development of GBC in both Japanese and Hungarian women and provided a rationale for further studies of genetic variation on the risk of GBC in Hungary Kimura et al. [56].

In 2006, a Chinese study investigated the potential risk associated with polymorphism in Cytochrome P450 17alphahydroxylases-C-(17,20)-lyase (CYP17), a key enzyme involved in the biosynthesis of sex hormones, in GBC. They found an association of the CYP17 MspA1 polymorphism with an increased risk of GBC, as well as biliary stones among overweight and diabetic individuals, which suggested interplay between 
genetic and hormonal risk factors in gallbladder disease Hou et al. [57]. Same year, a study conducted at our medical geneticist colleagues in India reported an association between glutathione S-transferase (GST) polymorphic variants (GSTT1, GSTM1, GSTP1, and GSTM3) with risk of gallbladder cancer. The GSTs are polymorphic supergene family of detoxification enzymes that are involved in the metabolism of numerous potential carcinogens.

Several allelic variants of polymorphic GSTs show impaired enzyme activity and are suspected to increase the susceptibility to various cancers. This was a first study showing val allele of GSTP1 to be associated with higher risk of gallbladder cancer in Indian population Pandey et al. [58]. Same group went on to publish another genetic risk factor associated GBC in 2007 by showing a polymorphic variant of $\mathrm{N}$-acetyl transferase2 (NAT2) which is also known as slow acetylator phenotype influencing the susceptibility of GBC Pandey et al. [59]. Same year also appeared a study from China that demonstrated that human oxoguanine glycosylase 1 (hOGG1) Ser326Cys polymorphism is associated with gallbladder. hOGG1 gene encodes a DNA glycosylase that is involved in excision repair of $8-0 \mathrm{H}-\mathrm{dG}$ (8-hydroxy-2-deoxyguanine) from oxidatively-damaged DNA cancer risk Jiao et al. [60]. To examine the relationship between genetic polymorphisms of cytochrome P450 1A1 (CYP1A1), glutathione S-transferase class mu (GSTM1), and tumour protein p53 (TP53) genes, and gallbladder cancer (GBC) risk, a case-control study conducted in Japan observed that Val allele of CYP1A1 Ile462Val polymorphism and the Pro allele of TP53 Arg72Pro polymorphism contributed to an increased risk of GBC among Japanese women and men, respectively Tsuchiya et al. [61].

Past decade has seen a growing body of data to demonstrate an association between various polymorphic variants of many cancers associated genes with risk of GBC. Majority of these studies came from researchers based in North India, some of important ones are those that report strong association between genetic variant of Tumor Necrosis Factor alpha (TNFA) and Interleukin 6 (IL6) Vishnoi et al. [62], A-204C of cholesterol 7alpha-hydroxylase (CYP7A1) Srivastava et al. [63], Cholecystokinin receptor A gene polymorphism Srivastava et al. [64] Interleukin-1 gene polymorphism Vishnoi et al. [65], Single nucleotide polymorphism in the ABCG8 transporter gene Srivastava et al. [66], toll-like receptor gene polymorphisms Srivastava et al. [67], and Caspase-8 polymorphisms, a gene that regulates apoptosis in cancer cells Srivastava et al. [68]. However, it must be noted that in spite of huge amount of efforts involved in these studies to study genetic risk factors, it is still unclear that which genetic pathways are actually important in order to make a person susceptible living in a geographically prone area for GBC such as North India.

All these genes demonstrated above which have been shown to be associated with GBC as risk factor belong to different signaling pathways, which further complicates the issue of making a coherent picture of all these genetic susceptibility factors. Many more studies have been published in recent past from different parts of the world showing one or other type of polymorphic variants of different genes involves in metabolism, signal transduction, drug-metabolizing enzymes related pathways, however, most of them are actually adding more complexity to the existing mechanistic insights about GBC.

\section{Targeted Therapy in GBC}

By mid 2000s, when increasing number of evidences were accumulating to demonstrate that overexpression of tyrosine kinase growth factor receptors such as ErbB-2, epidermal growth factor receptor (EGFR), and Met could play important roles in the development of many types of solid tumors, a group of investigators in Japan tested biliary tract carcinomas. With the aim of assessing the potential for novel chemotherapies targeting these receptors in $\mathrm{GBC}$, these investigators examined the overexpression of the tyrosine kinase receptor proteins was examined by immunohistochemistry in tumor tissues obtained from 89 gallbladder cancers. Positively stained tumours were further examined for gene amplification by fluorescence in situ hybridization.

Over expression of ErbB-2 and EGFR was found in 15.7\% and $8.1 \%$ respectively in gall bladder cancers gene amplification was present in $79 \%$ of these cancers. This study suggested the new adjuvant chemotherapies could be used in GBC in which ErbB-2 and EGFR are over expressed Nakazawa et al. [69]. Later a small Italian study also observed that a subgroup of patients with gallbladder carcinoma exhibited somatic mutations of EGFR in the tyrosine kinase domain that can elicit cell signals sustaining survival and proliferation. These group of investigators suggested that tumors might be further evaluated for their susceptibility to small-molecule inhibitor of RGFR for treatment Leone et al. [70]. Same year, a Chinese study reported that p53-VEGF (a marker for angiogenesis) pathway can regulate tumor angiogenesis in human gallbladder carcinoma. Combined analysis of p53 and VEGF expression might be useful for predicting the tumor vascularity of gallbladder cancer as well as it is indicative of targeting these tumors at advances stage with anti-VEGF inhibitors Tian et al. [71].

In order to better understand the mechanisms of tumor development and progression and to improve the prognosis of GBC patients, an small Austrian study observed an increased expression in HER2/neu which was significantly correlated with advanced $\mathrm{T}$ stages $(\mathrm{p}=0.02)$ Puhalla et al. [72]. Somatic mutations of the PIK3CA gene, which encodes the p110alpha catalytic subunit of phosphatidylinositol 3-kinase (PI3K), are frequent in various cancer types. The majority of mutations cluster at hotspots within exons 9 and 20, which encode the helical and kinase domains of p110alpha. A group based in Switzerland reported that somatic PIK3CA mutations contribute to the frequent activation of the PI3K/AKT pathway in a very small sub-set $(4 \%)$ of carcinomas of the gallbladder Riener et al. [73]. 
Realizing the lack of treatment options, besides surgical resection which also results in unfavorable prognosis for advanced GBC, a group of surgeons in Japan conducted a study to identify GBC-specific cellular targets that would form the basis for some therapeutic approaches for this disease. They identified a cell cycle-related gene, topoisomerase IIalpha (TOPO Ilalpha) as one of the highly upregulated gene in GBC tissue which they further confirmed as a potential chemotherapeutic target, because those cells strongly positive for TOPO IIalpha had shown increased sensitivity against etoposide, as well as doxorubicin and idarubicin Washiro et al. [74]. In subsequent year, another Japanese group demonstrated genomic instability due to amplification in Myc oncogene which resulted in specific amplification of EGFR and/or ERBB2 in GBC Ooi et al. [75]. A recently published in-vitro study also gives encouraging results using ac combination of Histone deacetylase inhibitor (SAHA) and repression of EZH2 by siRNA treatment, suggested a possible mechanism by which GBC cells but not normal cells showed an increased sensitivity to SAHA and indicates the efficacy of this new anticancer agent Yamaguchi et al. [76].

In a significant study to evaluate the response rate by Response Evaluation Criteria in Solid Tumors (RECIST) of targeted therapy in biliary cancers which included GBC, eligible patients (10 patients with GBC) were treated with bevacizumab (a VEGF inhibitor) and erlotinib (EGFR inhibitor) in combination with chemotherapy. Combination chemotherapy with bevacizumab and erlotinib showed clinical activity with infrequent grade 3 and 4 adverse effects in patients with advanced GBC. On the basis of preliminary molecular analysis, presence of a k-ras mutation may alter erlotinib efficacy. Though a small study in sample size (only 10 cases of GBC), results clearly warrant a larger study in future on a bigger cohort of GBC patients to investigate the efficacy of combination of bevacizumab and erlotinib as a potential therapeutic alternative in patients with advanced GBC Lubner et al. [77].

In an independent but encouraging small case report, the idea of using EGFR-tyrosine kinase inhibitor (TKI) in combination with conventional chemotherapy was tested to treat gallbladder cancer in United States based hospital. A 67 year old man with metastatic gallbladder cancer involving the liver and abdominal lymph nodes was treated with gemcitabine $\left(1000 \mathrm{mg} / \mathrm{m}^{2}\right.$, a commonly used chemotherapeutic agent for the treatment of GBC) on day 1 and 8 every 21 days as well as daily erlotinib (100 mg). After four cycles of therapy, the CA 19-9 normalized and a PET/CT showed a complete remission; this response was maintained by the end of 12 cycles of therapy. Gemcitabine was then discontinued and single agent erlotinib was continued as maintenance therapy.

The disease remains in good control 18 months after initiation of therapy, including 6 months on maintenance erlotinib. The only grade 3 toxicity was a typical EGFR-related skin rash. Because of the remarkable response to erlotinib plus gemcitabine, investigator performed tumor genotyping of the EGFR gene for response predicting mutations in exons 18, 19 and 21, which disclosed the wild-type genotype with no mutations. This case report demonstrates a patient with stage IV gallbladder cancer who experienced a rarely encountered complete, prolonged response after treatment with an oral EGFR-TKI plus chemotherapy. It is noticeable that this response occurred in the absence of an EGFR gene mutation. These observations should again emphasize the need of more clinical trials using EGFR-TKIs to treat GBC; and such trials should not select patients based on EGFR mutation status Mody et al. [78].

In an Italian study, investigators analyzed mutations, amplifications and over-expression of EGFR, HER2, and their molecular transducers in biliary tract cancers so that they could explore possibilities of combining standard therapies with or without molecular targeting. They found EGFR was expressed in $38.5 \%$ of patients with GBC. P-MAPK and p-Akt were also highly expressed in little less than $46 \%$ of GBC, indicating EGFR pathway activation. HER2 was overexpressed in $10 \%$ of GBCs with genomic amplification. They went on to conduct a preclinical in-vitro study using TGBC1-TKB, a GBC cell line (deleted on PTEN and negative for Her2 expression) for testing efficacy of drugs targeting these molecules, as single agents or in combination with gemcitabine. In this study, these investigators demonstrated that EGFR and HER2 pathways are suitable therapeutic targets for BTCs. The combination of gemcitabine with drugs targeting these pathways such as Gefitinib and Lapatinib (both of which are reversible selective inhibitors of the tyrosine kinase domain of EGFR) appeared to have encouraging results and thus warrants for further clinical studies for testing the expression levels and mutations in these signaling molecules in future clinical studies Pignochino et al. (2010).

While developing strategies to target GBC molecularly, it is important to understand the subtle changes at molecular genetic level between GBC and other related cancers which are albeit histologically similar, still anatomically different in origin of the tumor, such as cholangiocarcinomas arising from within the liver parenchyma, peri-hilar, or distal biliary tree, collectively known as Bile Tract Cancers (BTC). Although these tumors share an anatomic origin in the biliary system, there are important differences in disease behavior, molecular profiles, and sensitivity to therapy. Historically, GBC tends to be sensitive initially to chemotherapy but have a shorter overall survival compared with cholangiocarcinoma Eckel \& Schmid (2007). Traditionally, treatment for BTC has not taken into account the anatomic site of origin of the tumor or molecular profile and the mainstay of treatment is cytotoxic chemotherapy, but in the light of emerging molecular techniques especially availability of cost-effective next generation sequencing platforms, it will be worthwhile to have a detailed molecular genetic profiling of patients with GBC before considering a regimen of targeted therapy for GBC. 
Increasingly, molecular tests to detect changes at genetic level are being applied regularly (in developed countries where advance health facilities are available to common people) to assist in making therapeutic decisions in cancer treatment. HER2/NEU gene amplification, EGFR, and KRAS mutation testing are all used routinely in clinics to determine whether or not an individual will benefit from a treatment with specific targeted anti-cancer therapies McDermott \& Settleman (2009). Building on this paradigm, along with established molecularly targeted drugs, such as EGFR inhibitors, more emerging classes of drugs, such as BRAF inhibitors, are being tested at the earliest phases selectively in those patients who are believed to have the greatest potential for benefit based on their molecular genetic profiling Brower (2010). The fact that underlying tumor genetics may predict drug sensitivity- particularly in emerging classes of targeted anticancer agents, uncovering patterns of genetic changes within GBC becomes very critical in order to improve therapy as well as gaining insight into disease biology. In a very recent study conducted at Massachusetts General Hospital at Harvard Medical School in United States, almost 13\% of patients with GBC were identified with activating mutations in PIK3CA Deshpande et al. (2011). The presence of activating mutations in PIK3CA specifically in GBC has clinical implications in both the diagnosis of this cancer type, as well as the potential utility of targeted therapies such as PI3 kinase inhibitors.

Most recently, in a large whole-exome and targeted gene sequencing based study, first of its own kind, in gallbladder cancer patients in China, several recurrent mutations in erbB pathway were identified Li et al. [79]. In this study, most prominent genes with a significant frequency (false discovery rate (FDR) $<0.05$ ) of non-silent mutations included TP53 (47.1\%), KRAS (7.8\%) and ERBB3 (11.8\%). Moreover, ErbB signaling (including EGFR, ERBB2, ERBB3, ERBB4 and their downstream genes) is the most extensively mutated pathway, affecting $36.8 \%$ of the GBC samples. This study also demonstrated that mutations in genes in the ErbB pathway were associated with poor prognosis in individuals with GBC suggesting that patients harboring mutations in the ErbB pathway might benefit from targeted therapies presently used in the clinic or in development Li et al. [79]. Role of ErbB pathway in GBC was further confirmed in few other independent studies from different cohorts of patients. In one of such study, retrospectively conducted in the patients with GBC in the United States, HER2/neu blockade was found to be a promising treatment strategy to treat GBC patients who have gene amplification Javle et al. [80]. In another significant study conducted in Japan, in a large cohort of biliary tract cancers, activation of EGFR family genes such as ERBB2 and ERBB3, and inactivation of PTEN and TSC1 was found to be frequent in GBC. This study also reported frequent alterations of the TP53 and RB cell cycle modules in GBC Nakamura et al. [81].

Treatment options for patients with advanced un-resectable gallbladder cancer are limited owing to the lack of well-defined molecular targets. Complete surgical resection remains the only curative modality to treat patients with GBC, offering benefit only for patients with localized disease. In recent years, with identification of few molecular targets in GBC it is becoming apparent that development of effective therapies will be benefitted by focusing on the identification and targeting of genetic mutations specific to an individual patient. Further, using more sophisticated genomic tools on a larger cohorts of patients will not only help understand the pathobiology of this complex disease but also will be helpful in evaluating existing or novel therapeutic agents in clinical trials in the future.

\section{References}

1. Trajber HJ, Szego T, de Camargo HS, Mester M, Marujo WC, et al. (1982) Adenocarcinoma of the gallbladder in two siblings. Cancer 50(6): 1200-1203.

2. Weiss KM, Ferrell RE, Hanis CL, Styne PN (1984) Genetics and epidemiology of gallbladder disease in New World native peoples. Am J Hum Genet 36(6): 1259-1278.

3. Hecht F, Kuban DJ, Berger C, Hecht BK, Sandberg AA (1983) Adenocarcinoma of the gallbladder: chromosome abnormalities in a genetic form of cancer. Cancer Genet Cytogenet 8(3): 185-190.

4. Miyazaki K, Date K, Imamura S, Ogawa Y, Nakayama F (1989) Familial occurrence of anomalous pancreaticobiliary duct union associated with gallbladder neoplasms. Am J Gastroenterol 84(2): 176-181.

5. Tsuda T, Nakatani H, Tahara E, Sakamoto H, Terada M, et al. (1989) HST1 and INT2 gene coamplification in a squamous cell carcinoma of the gallbladder. Jpn J Clin Oncol 19(1): 26-29.

6. Tada M, Yokosuka O, Omata M, Ohto M, Isono K (1990) Analysis of ras gene mutations in biliary and pancreatic tumors by polymerase chain reaction and direct sequencing. Cancer 66(5): 930-935.

7. Kamel D, Pääkkö P, Nuorva K, Vähäkangas K, Soini Y (1993) p53 and c-erbB-2 protein expression in adenocarcinomas and epithelial dysplasias of the gall bladder. J Pathol 170(1): 67-72.

8. Roa I, Araya JC, Shiraishi T, Yatani R, Wistuba I, et al. (1993) DNA content in gallbladder carcinoma: a flow cytometric study of 96 cases. Histopathology 23(5): 459-464.

9. Takagi S, Naito E, Yamanouchi H, Ohtsuka H, Kominami R, et al. (1994) Mutation of the p53 gene in gallbladder cancer. Tohoku J Exp Med 172(3): 283-289.

10. Watanabe M, Asaka M, Tanaka J, Kurosawa M, Kasai M, et al. (1994) Point mutation of K-ras gene codon 12 in biliary tract tumors. Gastroenterology 107(4): 1147-1153.

11. Hanada K, Itoh M, Fujii K, Tsuchida A, Ooishi H, et al. (1996) K-ras and p53 mutations in stage I gallbladder carcinoma with an anomalous junction of the pancreaticobiliary duct. Cancer 77(3): 452-458.

12. Matsubara T, Sakurai Y, Sasayama Y, Hori H, Ochiai M, et al. (1996) K-ras point mutations in cancerous and noncancerous biliary epithelium in patients with pancreaticobiliary maljunction. Cancer 77: 1752-1757.

13. Tomono H, Nimura Y, Aono K, Nakashima I, Iwamoto T, et al. (1996) Point mutations of the c-Ki-ras gene in carcinoma and atypical epithelium associated with congenital biliary dilation. Am J Gastroenterol 91(6): 1211-1214

14. Saetta A, Lazaris AC, Davaris PS (1996) Detection of ras oncogene point mutations and simultaneous proliferative fraction estimation in gallbladder cancer. Pathol Res Pract 192(6): 532-540.

15. Iwase T, Nakazawa S, Yamao K, Yoshino J, Inui K, et al. (1997) Ras gene point mutations in gallbladder lesions associated with anomalous connection of pancreatobiliary ducts. Hepatogastroenterology. 44(17): $1457-1462$ 


\section{Cancer therapy \& Oncology International Journal}

16. Tanno S, Obara T, Fujii T, Mizukami Y, Shudo R, et al. (1998) Proliferative potential and K-ras mutation in epithelial hyperplasia of the gallbladder in patients with anomalous pancreaticobiliary ductal union. Cancer 83(2): 267-275.

17. Ajiki T, Onoyama H, Yamamoto M, Asaka K, Fujimori T, et al. (1996) p53 protein expression and prognosis in gallbladder carcinoma and premalignant lesions. Hepatogastroenterology 43(9): 521-526.

18. Fujii K, Yokozaki H, Yasui W, Kuniyasu H, Hirata M, et al. (1996) High frequency of p53 gene mutation in adenocarcinomas of the gallbladder Cancer Epidemiol Biomarkers Prev 5(6): 461-466.

19. Hanada K, Itoh M, Fujii K, Tsuchida A, Hirata M, et al. (1997) TP53 mutations in stage I gallbladder carcinoma with special attention to growth patterns. Eur J Cancer 33(7): 1136-1140.

20. Jonas S, Springmeier G, Tauber R, Wiedenmann B, Gessner R, et al. (1997) p53 hot-spot mutational analysis in advanced Western gallbladder carcinoma. World J Surg 21(7): 768-772.

21. Yokoyama N, Hitomi J, Watanabe H, Ajioka Y, Pruyas M, et al. (1998) Mutations of p53 in gallbladder carcinomas in high-incidence areas of Japan and Chile. Cancer Epidemiol Biomarkers Prev 7 (4): 297-301.

22. Wistuba II, Miquel JF, Gazdar AF, Albores-Saavedra J (1999) Gallbladder adenomas have molecular abnormalities different from those present in gallbladder carcinomas. Hum Pathol 30(1): 21-25

23. Wistuba II, Albores-Saavedra J (1999) Genetic abnormalities involved in the pathogenesis of gallbladder carcinoma. J Hepatobiliary Pancreat Surg 6(3): 237-244.

24. Yanagisawa N, Mikami T, Saegusa M, Okayasu I (2001) More frequent beta-catenin exon 3 mutations in gallbladder adenomas than in carcinomas indicate different lineages. Cancer Res 61(1): 19-22.

25. Yamato T, Sasaki M, Watanabe Y, Nakanuma Y (1999) Expression of MUC1 and MUC2 mucin core proteins and their messenger RNA in gall bladder carcinoma: an immunohistochemical and in situ hybridization study. J Pathol 188(1): 30-37.

26. Chang HJ, Kim SW, Kim YT, Kim WH (1999) Loss of heterozygosity in dysplasia and carcinoma of the gallbladder. Mod Pathol 12(8): 763769.

27. Hidaka E, Yanagisawa A, Sakai Y, Seki M, Kitagawa T, et al. (1999) Losses of heterozygosity on chromosomes $17 p$ and $9 p / 18 q$ may play important roles in early and advanced phases of gallbladder carcinogenesis. J Cancer Res Clin Oncol 125(8-9): 439-443.

28. Obara T, Tanno S, Fujii T, Izawa T, Mizukami Y, et al. (1999) Epithelial cell proliferation and gene mutation in the mucosa of gallbladder with pancreaticobiliary malunion and cancer. J Hepatobiliary Pancreat Surg 6(3): 229-236.

29. Yoshida T, Sugai T, Habano W, Nakamura S, Uesugi N, et al. (2000) Microsatellite instability in gallbladder carcinoma: two independent genetic pathways of gallbladder carcinogenesis. J Gastroenterol 35(10): 768-774.

30. Kim YT, Kim J, Jang YH, Lee WJ, Ryu JK, et al. (2001) Genetic alterations in gallbladder adenoma, dysplasia and carcinoma. Cancer Lett 169(1): 59-68.

31. Roa JC, Roa I, Correa P, Vo Q Araya JC, et al. (2005) Microsatellite instability in preneoplastic and neoplastic lesions of the gallbladder. J Gastroenterol 40(1): 79-86.

32. Saetta AA, Gigelou F, Papanastasiou PI, Koilakou SV, Kalekou-Greca $\mathrm{H}$, et al. (2006) High-level microsatellite instability is not involved in gallbladder carcinogenesis. Exp Mol Pathol 80(1): 67-71.

33. Nakayama K, Konno M, Kanzaki A, Morikawa T, Miyashita H, et al. (2001) Allelotype analysis of gallbladder carcinoma associated with anomalous junction of pancreaticobiliary duct. Cancer Lett 166(2): 135-141.

34. Wistuba II, Tang M, Maitra A, Alvarez H, Troncoso P, et al. (2001) Genome-wide allelotyping analysis reveals multiple sites of allelic loss in gallbladder carcinoma. Cancer Res 61(9): 3795-3800.

35. Saetta A, Lazaris AC, Michalopoulos NV, Davaris PS (2001) Genetic alterations involved in the development of gallbladder carcinomas from Greek patients. Hepatogastroenterology 48(41): 1284-1288.

36. Wistuba II, Ashfaq R, Maitra A, Alvarez H, Riquelme E, et al. (2002) Fragile histidine triad gene abnormalities in the pathogenesis of gallbladder carcinoma. Am J Pathol 160(6): 2073-2079.

37. Bloomston M, Kneile J, Butterfield M, Dillhoff M, Muscarella P, et al (2009) Coordinate loss of fragile gene expression in pancreatobiliary cancers: correlations among markers and clinical features. Ann Surg Oncol 16(8): 2331-2338.

38. Priya TP, Kapoor VK, Krishnani N, Agrawal V, Agarwal S (2009) Fragile histidine triad (FHIT) gene and its association with p53 protein expression in the progression of gall bladder cancer. Cancer Invest. 27(7): 764-773.

39. Takada M, Horita Y, Okuda S, Okumoto S, Samizo M, et al. (2002) Genetic analysis of xanthogranulomatous cholecystitis: precancerous lesion of gallbladder cancer? Hepatogastroenterology 49(46): 935-937.

40. Sessa F, Furlan D, Genasetti A, Billo P, Feltri M, et al. (2003) Microsatellite instability and p53 expression in gallbladder carcinomas. Diagn Mol Pathol 12(2): 96-102.

41. House MG, Wistuba II, Argani P, Guo M, Schulick RD, et al. (2003) Progression of gene hypermethylation in gallstone disease leading to gallbladder cancer. Ann Surg Oncol 10(8): 882-889.

42. Tang M, Baez S, Pruyas M, Diaz A, Calvo A, et al. (2004) Mitochondrial DNA mutation at the D310 (displacement loop) mononucleotide sequence in the pathogenesis of gallbladder carcinoma. Clin Cancer Res 10(3): 1041-1046.

43. Takahashi T, Shivapurkar N, Riquelme E, Shigematsu H, Reddy J, et al. (2004) Aberrant promoter hypermethylation of multiple genes in gallbladder carcinoma and chronic cholecystitis. Clin Cancer Res 10(18 Pt 1): 6126-6133.

44. Roa JC, Anabalón L, Roa I, Melo A, Araya JC, et al. (2006) Promoter methylation profile in gallbladder cancer. J Gastroenterol 41(3): 269275 .

45. Riquelme E, Tang M, Baez S, Diaz A, Pruyas M, et al. (2007) Frequent epigenetic inactivation of chromosome 3 p candidate tumor suppressor genes in gallbladder carcinoma. Cancer Lett 250(1): 100-106.

46. Saetta AA, Papanastasiou P, Michalopoulos NV, Gigelou F, Korkolopoulou $\mathrm{P}$, et al. (2004) Mutational analysis of BRAF in gallbladder carcinomas in association with K-ras and p53 mutations and microsatellite instability. Virchows Arch 445(2): 179-182.

47. Singh MK, Pandey UB, Ghoshal UC, Srivenu I, Kapoor VK, et al. (2004) Apolipoprotein $\mathrm{B}-100 \mathrm{XbaI}$ gene polymorphism in gallbladder cancer Hum Genet 114(3): 280-283

48. Singh MK, Chetri K, Pandey UB, Kapoor VK, Mittal B, et al. (2004) Mutational spectrum of K-ras oncogene among Indian patients with gallbladder cancer. J Gastroenterol Hepatol 19(8): 916-921.

49. Tadokoro H, Shigihara T, Ikeda T, Takase M, Suyama M (2007) Two distinct pathways of p16 gene inactivation in gallbladder cancer. World J Gastroenterol 13(47): 6396-6403.

50. Nagahashi M, Ajioka Y, Lang I, Szentirmay Z, Kasler M, et al. (2008) Genetic changes of p53, K-ras, and microsatellite instability in gallbladder carcinoma in high-incidence areas of Japan and Hungary. World J Gastroenterol 14(1): 70-75. 


\section{Cancer therapy \& Oncology International Journal}

51. Alvarez H, Corvalan A, Roa JC, Argani P, Murillo F, et al. (2008) Serial analysis of gene expression identifies connective tissue growth facstor expression as a prognostic biomarker in gallbladder cancer. Clin Cancer Res 14(9): 2631-2638.

52. Wang JW, Peng SY, Li JT, Wang Y, Zhang ZP, et al. (2009) Identification of metastasis-associated proteins involved in gallbladder carcinoma metastasis by proteomic analysis and functional exploration of chloride intracellular channel 1. Cancer Lett 281(1):71-81.

53. Miller G, Socci ND, Dhall D, D'Angelica M, DeMatteo RP, et al. (2009) Genome wide analysis and clinical correlation of chromosomal and transcriptional mutations in cancers of the biliary tract. J Exp Clin Cancer Res 28: 62.

54. Priya TP, Kapoor VK, Krishnani N, Agrawal V, Agrawal S (2010) Role of E-cadherin gene in gall bladder cancer and its precursor lesions. Virchows Arch 456(5): 507-514.

55. Tsuchiya Y, Sato T, Kiyohara C, Yoshida K, Ogoshi K, et al. (2002) Genetic polymorphisms of cytochrome P450 1A1 and risk of gallbladder cancer. J Exp Clin Cancer Res 21(1): 119-124.

56. Kimura A, Tsuchiya Y, Lang I, Zoltan S, Nakadaira H, et al. (2008) Effect of genetic predisposition on the risk of gallbladder cancer in Hungary. Asian Pac J Cancer Prev 9(3): 391-396.

57. Hou L, Xu J, Gao YT, Rashid A, Zheng SL, et al. (2006) CYP17 MspA1 polymorphism and risk of biliary tract cancers and gallstones: a population-based study in Shanghai, China. Int J Cancer 118(11) 2847-2853

58. Pandey SN, Jain M, Nigam P, Choudhuri G, Mittal B (2006) Genetic polymorphisms in GSTM1, GSTT1, GSTP1, GSTM3 and the susceptibility to gallbladder cancer in North India. Biomarkers 11(3): 250-261.

59. Pandey SN, Modi DR, Choudhuri G, Mittall B (2007) Slow acetylator genotype of N-acetyl transferase2 (NAT2) is associated with increased susceptibility to gallbladder cancer: the cancer risk not modulated by gallstone disease. Cancer Biol Ther 6(1): 91-96.

60. Jiao X, Huang J, Wu S, Lv M, Hu Y et al. (2007) hOGG1 Ser326Cys polymorphism and susceptibility to gallbladder cancer in a Chinese population. Int J Cancer 121(3): 501-505.

61. Tsuchiya Y, Kiyohara C, Sato T, Nakamura K, Kimura A, et al. (2007) Polymorphisms of cytochrome P450 1A1, glutathione S-transferase class mu, and tumour protein p53 genes and the risk of developing gallbladder cancer in Japanese. Clin Biochem 40(12): 881-886.

62. Vishnoi M, Pandey SN, Choudhury G, Kumar A, Modi DR, et al. (2007) Do TNFA -308G/A and IL6 -174 G/C gene polymorphisms modulate risk of gallbladder cancer in the north Indian population? Asian Pac J Cancer Prev 8(4): 567-572.

63. Srivastava A, Pandey SN, Choudhuri G, Mittal B (2008) Role of genetic variant A-204C of cholesterol 7alpha-hydroxylase (CYP7A1) in susceptibility to gallbladder cancer. Mol Genet Metab 94(1): 83-89.

64. Srivastava A, Pandey SN, Dixit M, Choudhuri G, Mittal B (2008) Cholecystokinin receptor A gene polymorphism in gallstone disease and gallbladder cancer. J Gastroenterol Hepatol 23(6): 970-975.

65. Vishnoi M, Pandey SN, Choudhuri G, Mittal B (2008) IL-1 gene polymorphisms and genetic susceptibility of gallbladder cancer in a north Indian population. Cancer Genet Cytogenet 186(2): 63-68.
66. Srivastava A, Tulsyan S, Pandey SN, Choudhuri G, Mittal B (2009) Single nucleotide polymorphism in the ABCG8 transporter gene is associated with gallbladder cancer susceptibility. Liver Int 29(6): 831-837.

67. Srivastava K, Srivastava A, Kumar A, Mittal B (2010) Significant association between toll-like receptor gene polymorphisms and gallbladder cancer. Liver Int 30(7): 1067-1072.

68. Srivastava K, Srivastava A, Mittal B (2010) Caspase-8 polymorphisms and risk of gallbladder cancer in a northern Indian population. Mol Carcinog 49(7): 684-692.

69. Nakazawa K, Dobashi Y, Suzuki S, Fujii H, Takeda Y, et al. (2005) Amplification and overexpression of c-erbB-2, epidermal growth factor receptor, and c-met in biliary tract cancers. J Pathol 206(3): 356-365.

70. Leone F, Cavalloni G, Pignochino Y, Sarotto I, Ferraris R, et al. (2006) Somatic mutations of epidermal growth factor receptor in bile duct and gallbladder carcinoma. Clin Cancer Res 12(6): 1680-1685.

71. Tian Y, Ding RY, Zhi YH, Guo RX, Wu SD (2006) Analysis of p53 and vascular endothelial growth factor expression in human gallbladder carcinoma for the determination of tumor vascularity. World Gastroenterol 12(3): 415-419.

72. Puhalla H, Wrba F, Kandioler D, Lehnert M, Huynh A, et al. (2007) Expression of p21(Wafl/Cip1), p57(Kip2) and HER2/neu in patients with gallbladder cancer. Anticancer Res 27(3B): 1679-1684.

73. Riener MO, Bawohl M, Clavien PA, Jochum W (2008) Rare PIK3CA hotspot mutations in carcinomas of the biliary tract. Genes Chromosomes Cancer 47(5): 363-367.

74. Washiro M, Ohtsuka M, Kimura F, Shimizu H, Yoshidome H, et al (2008) Upregulation of topoisomerase IIalpha expression in advanced gallbladder carcinoma: a potential chemotherapeutic target. J Cancer Res Clin Oncol 134(7): 793-801.

75. Ooi A, Suzuki S, Nakazawa K, Itakura J, Imoto I, et al. (2009) Gene amplification of Myc and its coamplification with ERBB2 and EGFR in gallbladder adenocarcinoma. Anticancer Res 29(1): 19-26.

76. Yamaguchi J, Sasaki M, Sato Y, Itatsu K, Harada K, et al. (2010) Histone deacetylase inhibitor (SAHA) and repression of EZH2 synergistically inhibit proliferation of gallbladder carcinoma. Cancer Sci 101(2): 355362.

77. Lubner SJ, Mahoney MR, Kolesar JL, Loconte NK, Kim GP, et al. (2010) Report of a multicenter phase II trial testing a combination of biweekly bevacizumab and daily erlotinib in patients with unresectable biliary cancer: a phase II Consortium study. J Clin Oncol 28(21): 3491-3497.

78. Mody K, Strauss E, Lincer R, Frank RC (2010) Complete response in gallbladder cancer to erlotinib plus gemcitabine does not require mutation of the epidermal growth factor receptor gene: a case report. BMC Cancer 10: 570.

79. Li M, Zhang Z, Li X, Ye J, Wu X, et al. (2014) Whole-exome and targeted gene sequencing of gallbladder carcinoma identifies recurrent mutations in the ErbB pathway. Nat Genet 46(8): 872-876.

80. Javle M, Churi C, Kang HC, Shroff R, Janku F, et al. HER2/neu-directed therapy for biliary tract cancer. J Hematol Oncol 8: 58.

81. Nakamura H, Arai Y, Totoki Y, Shirota T, Elzawahry A, et al. (2015) Genomic spectra of biliary tract cancer. Nat Genet 47(9): 1003-1010. 


\section{Your next submission with Juniper Publishers} will reach you the below assets

- Quality Editorial service

- Swift Peer Review

- Reprints availability

- E-prints Service

- Manuscript Podcast for convenient understanding

- Global attainment for your research

- Manuscript accessibility in different formats

( Pdf, E-pub, Full Text, Audio)

- Unceasing customer service

Track the below URL for one-step submission https://juniperpublishers.com/online-submission.php 